\title{
Implications of HER2-Targeted Therapy on Extent of Surgery for Early-Stage Breast Cancer
}

\author{
Hamza Aziz, MD¹, P. Kelly Marcom, MD², and E. Shelley Hwang, MD, MPH ${ }^{1}$ \\ ${ }^{1}$ Division of Surgical Oncology, Department of Surgery, Duke University Medical Center, Durham, NC; ${ }^{2}$ Division of \\ Medical Oncology, Department of Medicine, Duke University Medical Center, Durham, NC
}

Over the past three decades, our understanding of the molecular underpinnings of breast cancer has improved at an unprecedented rate. During this same period, mammographic screening has led to a doubling in the incidence of early-stage breast cancers and an eightfold increase in the incidence of DCIS. ${ }^{1}$ This has led to tremendous interest in understanding the biology of early-stage breast cancers, including DCIS and tailoring both local and systemic treatment to the molecular, histologic, and morphologic features that drive tumor behavior.

HER2, a transmembrane receptor with tyrosine kinase activity, is overexpressed at the mRNA and/or protein level in approximately $20 \%$ of early-stage breast cancer. Before the widespread use of HER2-targeted therapy, primarily with the monoclonal antibody trastuzumab, overexpression of HER2 was clearly associated with high recurrence rates and increased mortality. ${ }^{2}$ The HER2-positive subtype confers a high risk of local failure independent of whether patients were treated with breast-conserving therapy (BCT) or mastectomy. An extensive analysis of 10-year local recurrence-free survival (LRFS) showed that the HER2 subtype had a LRFS of $83 \%$ after mastectomy and $79 \%$ after BCT compared with $92 \%$ for both mastectomy and $\mathrm{BCT}$ in the Luminal A subtype. ${ }^{3}$ Important to note, however, is that this study reported on patients diagnosed from 1986 to 1992 , before use of trastuzumab.

In the preceding article, Dr. Tot examined 203 consecutive cases of newly diagnosed invasive breast carcinomas measuring $<10 \mathrm{~mm}$ in size during the period 2007-2013 at a

(C) Society of Surgical Oncology 2015

First Received: 1 March 2015;

Published Online: 17 March 2015

E. S. Hwang, MD, MPH

e-mail: shelley.hwang@duke.edu single institution, including 23 cases of HER2-positive disease. A validation cohort of 80 cases from 1996 to 1998 also was analyzed. In both sets of samples, HER2-positive breast cancers were found to be more often associated with a higher component of diffuse DCIS around the invasive tumor ( $p<0.0001)$, more likely to be of higher grade $(p=0.0004)$, and have lymphovascular invasion $(p=0.0026)$ compared with tumors lacking HER2 overexpression. Although no multivariable analysis or recurrence data were provided, the author postulates a causal role of extensive DCIS for HER2positive breast cancer recurrence.

The presence of an extensive intraductal component (EIC) has been recognized to be associated with a higher local recurrence rate following $\mathrm{BCT}$, long before genomic subtyping of tumors. ${ }^{4,5}$ However, the impact of EIC on local outcomes has been shown to be largely abrogated when free margins are achieved and when adjusted for age at diagnosis. ${ }^{5-7}$ In the recently published ASCO/ASTRO guidelines, an extensive meta-analysis failed to support that larger margins were indicated for any tumor phenotype, including the HER2-positive subtype. ${ }^{8}$ Moreover, HER2-positive breast cancers are biologically more aggressive independent of EIC, as is well demonstrated in Dr. Tot's own data. In the absence of outcomes in Dr. Tot's study, it is difficult to recommend any change in surgical treatment based on these observations alone, but an awareness of the association of HER2-positive disease with extensive involvement should highlight the importance of careful preoperative evaluation in order to accurately determine disease extent.

Concurrent with recent efforts to decrease surgical morbidity, there has been an emerging recognition of the importance of systemic treatments to improve locoregional control. HER2-positive disease has been a true success of targeted therapy, with trastuzumab improving cancer mortality in HER2-overexpressing tumors by $33 \%{ }^{9}$ Moreover, local recurrence has also been shown to be 
significantly reduced, from 3 to $1.6 \%$ at a median followup of 1 year. ${ }^{9}$ Recent, retrospective, single-institution data support this benefit and have shown significant improvement in LRFS after both mastectomy and BCT with trastuzumab therapy. ${ }^{10,11}$

Moreover, an expanded use of trastuzumab-based systemic therapy has been prompted by multiple retrospective studies showing a higher distant recurrence risk for HER2positive $\mathrm{T} 1 \mathrm{~b} / \mathrm{c}$ stage I cancers than estimated from conventional staging. ${ }^{12}$ Consequently, guidelines now recommend trastuzumab for most HER2-positive lesions $>5 \mathrm{~mm}$ and many are considering treatment for cancers below this threshold as well. ${ }^{13,14}$ A recent study of a 12-week course of adjuvant paclitaxel and trastuzumab in node-negative, earlystage tumors (almost half of which were $<1 \mathrm{~cm}$ ) reported a 3year, disease-free survival of $98.7 \%$, indicating the effectiveness of chemotherapy and trastuzumab, albeit a relatively less intense regimen, for these cancers. ${ }^{15}$ Dr. Tot's data provide some insight into the disconnect between conventional assessment of tumor size and prognosis in HER2positive disease and indicate that in situ and invasive disease distribution should be examined in prospective trials in this disease subset.

Relevant to the issue of trastuzumab for DCIS, NSABP43 is a phase III clinical trial for women with HER2/neupositive DCIS without invasion treated with lumpectomy, randomized to radiation with two doses of trastuzumab versus radiation without trastuzumab, with the primary endpoint of ipsilateral breast cancer recurrence. ${ }^{16}$ This study has almost completed accrual, and results from this trial are awaited with interest, as the evidence could support an additional option in the armamentarium for HER2/ neu-positive DCIS.

Future advances in the care of women with early HER2positive disease will increasingly rely on tailoring our approach based on molecular and histologic profiles while excising only what is required to provide optimal locoregional control for the patient. Given the expanding role of trastuzumab and other HER2-targeted therapies even for small tumors, as well as the impact of systemic treatments on local recurrence as well as distant recurrence, the role of extensive surgery is likely to play a diminished role as the primary determinant of long-term local disease control. Thus, future treatment paradigms will require a thoughtful implementation of a multidisciplinary approach, even for the earliest of cancers.

\section{REFERENCES}

1. Bleyer A, Welch HG. Effect of three decades of screening mammography on breast-cancer incidence. N Engl J Med. 2012;367:1998-2005.
2. Arteaga CL, Sliwkowski MX, Osborne CK, et al. Treatment of HER2-positive breast cancer: current status and future perspectives. Nat Rev Clin Oncol. 2012;9:16-32.

3. Voduc KD, Cheang MC, Tyldesley S, et al. Breast cancer subtypes and the risk of local and regional relapse. J Clin Oncol. 2010;28:1684-91.

4. Jacquemier J, Kurtz JM, Amalric R, et al. An assessment of extensive intraductal component as a risk factor for local recurrence after breast-conserving therapy. Br J Cancer. 1990;61: 873-6.

5. Wazer DE, Schmidt-Ullrich RK, Ruthazer R, et al. The influence of age and extensive intraductal component histology upon breast lumpectomy margin assessment as a predictor of residual tumor. Int J Radiat Oncol Biol Phys. 1999;45:885-91.

6. Douglas-Jones AG, Logan J, Morgan JM, et al. Effect of margins of excision on recurrence after local excision of ductal carcinoma in situ of the breast. J Clin Pathol. 2002;55:581-6.

7. Hurd TC, Sneige N, Allen PK, et al. Impact of extensive intraductal component on recurrence and survival in patients with stage I or II breast cancer treated with breast conservation therapy. Ann Surg Oncol. 1997;4:119-24.

8. Moran MS, Schnitt SJ, Giuliano AE, et al. Society of Surgical Oncology-American Society for Radiation Oncology consensus guideline on margins for breast-conserving surgery with wholebreast irradiation in stages I and II invasive breast cancer. J Clin Oncol. 2014;32:1507-15.

9. Piccart-Gebhart MJ, Procter M, Leyland-Jones B, et al. Trastuzumab after adjuvant chemotherapy in HER2-positive breast cancer. N Engl J Med. 2005;353:1659-72.

10. Kiess AP, McArthur HL, Mahoney K, et al. Adjuvant trastuzumab reduces locoregional recurrence in women who receive breast-conservation therapy for lymph node-negative, human epidermal growth factor receptor 2-positive breast cancer. Cancer. 2012;118:1982-8.

11. Lanning RM, Morrow M, Riaz N, et al. The effect of adjuvant trastuzumab on locoregional recurrence of human epidermal growth factor receptor 2-positive breast cancer treated with mastectomy. Ann Surg Oncol. 2015. doi:10.1245/s10434-0144321-2.

12. Gonzalez-Angulo AM, Litton JK, Broglio KR, et al. High risk of recurrence for patients with breast cancer who have human epidermal growth factor receptor 2-positive, node-negative tumors $1 \mathrm{~cm}$ or smaller. J Clin Oncol. 2009;27:5700-6.

13. Gradishar WJ, Anderson BO, Blair SL, et al. Breast Cancer Version 3.2014. J Natl Comprehens Cancer Netw. 2014; 12: 542-90.

14. Goldhirsch A, Winer EP, Coates AS, et al. Personalizing the treatment of women with early breast cancer: highlights of the St Gallen International Expert Consensus on the Primary Therapy of Early Breast Cancer 2013. Ann Oncol. 2013; 24: 2206-23.

15. Tolaney SM, Barry WT, Dang CT, et al. Adjuvant paclitaxel and trastuzumab for node-negative, HER2-positive breast cancer. N Engl J Med 2015; 372: 134-141.

16. Siziopikou KP, Anderson SJ, Cobleigh MA, et al. Preliminary results of centralized HER2 testing in ductal carcinoma in situ (DCIS): NSABP B-43. Breast Cancer Res Treat. 2013;142:41521. 